\title{
Influence d'une réduction du taux d'azote indifférencié sur le niveau d'ingestion alimentaire et les performances de croissance du porc femelle, selon la concentration et la nature des substrats énergétiques dans le régime.
}

\author{
J. NOBLET, Y. HENRY et D. BOURDON
}

Avec la collaboration technique de Charital BLONDEI, Régine CALMES, G. CONSEIL, D. CARON, R. LEVREL

\author{
Station de Recherches sur l'Élevage des Porcs \\ Centre de Rennes-St-Gilles, I.N.R.A., \\ 35590 L'Hermitage (France)
}

\section{Résumé}

Une expérience a été réalisée sur 63 porcs femelles de race Large-White entre 25 et roo $\mathrm{kg}$ de poids vif ,afin d'étudier l'influence d'une réduction du taux d'azote indifférencié dans la ration sur le niveau de consommation, les performances de croissance et les caractéristiques de composition corporelle, selon la concentration en énergie et la nature des substrats énergétiques. Sur les 6 lots de 9 animaux, 3 reçoivent des rations normalement pourvues en matières azotées différant, soit par la concentration en énergie (lot : I maïs-tourteau de soja et lot 3 : mais-tourteau de soja dilué avec de la vermiculite), soit par la nature des substrats énergétiques, à concentrations énergétiques comparables : lot 3 et lot 5 (orge-tourteau de soja). Dans les 3 autres lots (lots 2,4 et 6 ), on procède, pour chacun des 3 régimes précédents à une réduction d'environ 25 p. Ioo du taux de matières azotées, avec supplémentation en acides aminés indispensables (L-lysine, L-tryptophane, L-thréonine), de façon à assurer la couverture des besoins des animaux Ce schéma expérimental a été complété par un septième lot, afin de contrôler l'efficacité de la supplémentation en thréonine d'un régime orge-tourteau de soja à taux azoté réduit. Afin d'étudier l'influence de la concentration en énergie et de la nature des substrats énergétiques sur l'utilisation digestive de l'énergie et des matières azotées, il a été procédé à une comparaison des régimes $\mathrm{r}, 3$ et 5 en cages à métabolisme, à raison de 4 animaux par traitement.

La dilution du régime maîs-tourteau de soja par la vermiculite s'accompagne d'une réduction significative de la digestibilité de l'ensemble des constituants du régime (o,28 point pour I p. Ioo de vermiculite, pour l'énergie). De la même façon, le remplacement du maîs par l'orge entraîne une diminution importante de l'utilisation digestive des nutriments $(-9,8$ p. Ioo pour l'énergie).

L'introduction de vermiculite (lot 3 ) dans le régime maïs-soja (lot $\mathrm{I}$ ) ou le remplacement du maïs par l'orge (lot 5) s'accompagnent d'une augmentation de la quantité d'aliment consommée (kg aliment), la consommation d'Energie Digestible (E.D.) restant inférieure, et d'un maintien de la vitesse de croissance. Toutefois, la réduction de la concentration énergétique du régime ne modifie pratiquement pas les caractéristiques de composition corporelle dans le cas de la dilution du régime maïs-soja par la vermiculite., alors que l'adiposité des carcasses est significativement abaissée après remplacement du maīs par l'orge.

A la suite d'une réduction du taux azoté avec supplémentation en acides aminés limitants on observe une surconsommation d'aliment, marquée $(+6 \mathrm{p}$. Ioo), faible $(+2 \mathrm{p}$. Ioo) ou nulle, 
respectivement pour les régimes mais-soja, orge-orge et mais-soja dilué avec de la vermiculite, sans que les vitesses de croissance soient modifiées significativement. Il s'ensuit un accroissement đu coût énergétique du gain ainsi qu'une augmentation de l'adiposité des carcasses, celle-ci étant importante dans le cas des régimes orge-soja.

Enfin, la thréonine constitue, après la lysine, l'acide aniné limitant secondaire, d'un régime orge-tourteau de soja, à taux azoté réduit.

\section{Introduction}

Dans un travail antérieur (NoBLET et HENRY, I977), nous avions montré que les quantités d'aliment ingérées par le porc, nourri à volonté, sont influencées à la fois par la concentration en substrats énergétiques du régime, le taux de matières azotées et l'équilibre de ces dernières en acides aminés. En particulier, lorsque 1'apport des acides aminés indispensables les plus limitants est maintenu constant, la réponse du porc à une réduction du taux de matières azotées, semble être modifiée par la concentration en substrats énergétiques et/ou leur nature (céréale utilisée). Il nous a donc paru intéressant de poursuivre cette étude en essayant de dissocier ces deux effets:

- d'une part, en comparant un régime relativement riche en énergie (association maîs - tourteau de soja) avec un régime de même type mais dilué en énergie par incorporation d'un diluant inerte, la vermiculite (effet propre de la concentration en substrats énergétiques),

- d'autre part, en comparant le régime dilué en énergie avec un régime à b ase d'orge - tourteau de soja, à concentration équivalente en énergie digestible (effet propre de la nature du substrat énergétique).

- Dans les deux cas en étudiant la variation de réponse selon la concentration en azote indifférencié dans des régimes équilibrés par ailleurs en acides aminés indispensables.

Une expérience a ainsi été réalisée sur des porcs femelles entre 25 et roo $\mathrm{kg}$ de poids vif, portant sur l'étude des effets comparés de la concentration et de la nature des substrats énergétiques sur le niveau d'ingestion alimentaire et les résultats de croissance et de composition corporelle, selon le taux d'azote indifférencié. Sur la base des conclusions de l'étude précédente, le travail avait en outre pour objet de contrôler l'efficacité de la supplémentation en thréonine sous forme libre dans un régime à base d'orge à taux azoté réduit.

\section{Matériel et méthodes}

\section{I. - Expérience en lots}

Soixante-trois porcelets femelles de race Large-White, d'un poids vif moyen initial de $26,2 \mathrm{~kg}$ sont répartis, à l'isstue d'une période préexpérimentale commune de 2 semaines, en 7 lots de 9 animaux, suivant un dispositif en blocs complets équilibrés, compte tenu du poids vif et de 1'âge.

Comme dans l'essai précédent (NoBlet et HENRY, I977), les lots d'animaux sont répartis en 2 groupes différart par la nature de la céréale utilisée dans le 
régime : le prenier (lots $I, 2,3$ et 4) reçoit des régimes à base de maïs, céréale riche en énergie, le deuxième (lots 5,6 et 7 ) des régimes à base d'orge, céréale de valeur énergétique moyenne, en association avec le tourteau de soja (tab1. I).

Les régimes des lots I et 5 sont normalement pourvus en matières azotées et en acides aminés, suivant les recommandations habituelles (HENRy, PIoN et RERAT, I 976). Leurs teneurs en matières azotées digestibles (M.A.D.), relativement à 1'énergie digestible (F.D.), sont ajustées à 44 et $39 \mathrm{~g} /$ Mcal respectivement jusqu'à $50 \mathrm{~kg}$ de poids vif (période dite de "croissance ") et au-delà (période dite de "finition "), sur la base des valeurs d'utilisation digestive des matières azotées de 87 et 83 p. IOO, respectivement pour les régimes à base de maïs et d'orge (HENRY, I976).

Dans les régimes 2 et 6 , on procède à une réduction d'environ $25 \mathrm{p}$. Ioo de la teneur en matières azotées globales, compensée par un apport supplémentaire des acides aminés limitants primaire (L-Lysine) et secondaire (L-tryptophane dans le lot 2 à base de maïs, L-thréonine et L-tryptophane dans le lot 5 , à base d'orge), pour satisfaire les besoins en acides aminés indispensables.

Un diluant inerte (vermiculite) est introduit dans le régime 3 afin d'abaisser la concentration en E.I. du régime à base de maïs au niveau de la concentration des régimes à base d'orge (lots 5 à 7 ). Le rapport M.A.D./F.D. est ajusté à la même valeur que celle prévue pour les régimes témoins I et 5 , compte tenu de l'effet dépressif prévisible de la vermiculite sur l'utilisation digestive de l'énergie et des matières azotées (HENRy et ETIENNE, I969). Le rapport M.A.D./E.D. est réduit de $25 \mathrm{p}$. Ioo dans le régime 4 , dans les mêmes conditions que pour les régimes 2 et 6 avec un apport supplémentaire de lysine et de tryptophane. Une addition de thréonine est également pratiquée de manière à corriger une indisponibilité éventuelle de cette dernière en présence de vermiculite.

Enfin, le lot 6 est comparé à un septième lot, recevant un régime à base d'orge, à taux azoté réduit et non supplémenté en thréonine, pour s'assurer de l'efficacité d'un apport supplémentaire de thréonine dans les conditions expérimentales ainsi décrites. La composition des régimes expérimentaux est rapportée dans le tableau $\mathrm{I}$.

Les animaux sont élevés en loges individuelles. L'aliment, présenté sous forme de granulés de $5 \mathrm{~mm}$ de diamètre, est distribué à volonté, les consommations étant enregistrées quotidiennement. L es pores sont pesés toutes les deux semaines. A IOO $\pm z \mathrm{~kg}$ de poids vif, ils sont abattus et, après un ressuyage de 24 heures, les carcasses sont soumises à la découpe parisienne.

Les résultats de consommation et de croissance pour les périodes $25-50 \mathrm{~kg}$ et 25 -IOo $\mathrm{kg}$ de poids vif, ainsi que les caractéristiques de composition corporelle, dans les lots I à 6 , sont traités par l'analyse de variance, avec séparation des effets principaux (concentration en substrats énergétiques, réduction du taux d'azote indifférencié, nature des substrats énergétiques, nature de la céréale), et des effets d'interaction. Les données du lot 7 sont comparées directement à celles du lot 6 .

\section{II. - Essai de digestibilité}

Afin de préciser l'effet de la dilution énergétique sur l'utilisation digestive de l'énergie et des matières azotées, dans les régimes normalement pourvus en matières azotées (lots I, 3 et 5 ), un essai de digestibilité a éré conduit sur I 2 porcs mâles castrés, répartis en 4 blocs de 3 animaux homogènes .Au sein de chaque bloc, les animaux sont affectés au hasard à l'un des 3 traitements. Deux collectes 


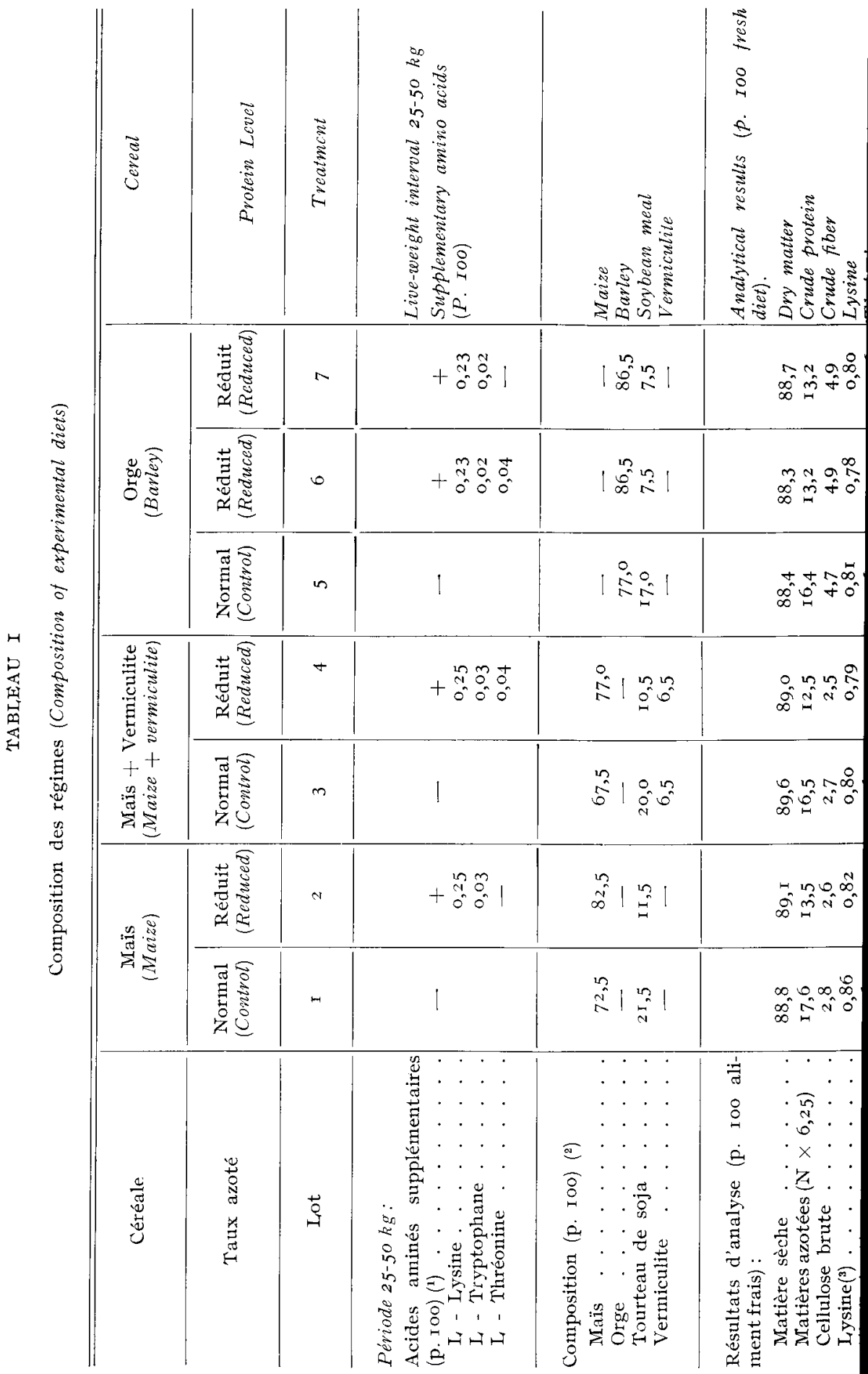


d'une durée de ro jours chacune sont effectuées sur le même animal, l'une correspondant à l'aliment utilisé jusqu'à $50 \mathrm{~kg}$ de poids vif (poids vif moyen : $35,5 \mathrm{~kg}$ ), l'autre correspondant à 1'aliment utilisé au delà de $50 \mathrm{~kg}$ (poids moyen : $57,2 \mathrm{~kg}$ ). Dans les 3 traitements, les porcs reçoivent la même quantité d'aliment pendant toute la durée de la collecte, soit I, 4 et I, $8 \mathrm{~kg}$, respectivement au cours des 2 périodes. Les modalités expérimentales concernant l'adaptation des animaux aux cages de digestibilité et la collecte des excréta sont identiques à celles décrites par HeNRY et BOURDON (I973).

Les résultats sont également traités par l'analyse de variance, avec séparation des effets principaux (période, concentration ou nature des substrats énergétiques, nature de la céréale) et des effets d'interaction.

\section{Résultats}

Sur les 63 animaux de l'expérience en lots, 3 ont dû être éliminés avant la fin de l'essai : un dans le lot 2, un dans le lot 5 (rachitisme), un dans le lot 7 (ulcère gastrique). Les résultats des deux expériences sont rapportés dans les tableaux 2,3 et 4 .

I. - Influence de la concentration en énergie et de la nature des substrats énergétiques sur l'utilisation digestive de l'énergie et des matières azotées (tab1. 2)

La comparaison des résultats obtenus au cours des deux périodes d'observation fait apparaitte une augmentation significative d'environ un point des cofficients d'utilisation digestive apparents de la matière organique (CUD MO) et de l'énergie (CUD E) lorsque le poids vif moyen des animaux s'élève de 35 à $58 \mathrm{~kg}$, quel que soit le régime, tandis que l'utilisation digestive des matières azotées n'est pas modifiée.

En dehors de son effet de dilution du contenu énergétique du régime, 1'introduction de la vermiculite entraîne une diminution significative de la digestibilité de l'ensemble des constituants du régime : soit pour la matière organique, l'énergie et les matières azotées, respectivement $0,25,0,28$ et 0,76 point pour I p. Ioo d'augmentation de la vermiculite dans la ration. Ainsi, une réduction de 6,8 p. xoo. de la teneur en énergie brute par addition d'un diluant inerte, se traduit par une diminution plus importante de la teneur en énergie digestible $(-8,0$ p. Ioo). L'effet de la vermiculite est cependant plus marqué sur 1'utilisation digestive des matières azotées (- 4,5 p. IOO) que sur celle de l'ensemble de la matière organique ( $\mathrm{r}, 6 \mathrm{p}$. IOo). Il en résulte un rapport $\mathrm{MAD} / \mathrm{ED}$ plus faible dans le régime contenant de la vermiculite (lots 3 et 4 ) : 44,4 en "croissance " et 39,2 en "finition ", contre, respectivement, 46,8 et $4 \mathrm{I}, 2$.

Le remplacement du maïs par l'orge se traduit également par une diminution importante de l'utilisation digestive des nutriments, soit respectivement, 8, 5, 9,8 et 7,6 p. Ioo pour la matière organique, l'énergie et les matières azotées.

Le cofficient de rétention azotée paraît modifié par l'origine des protéines de la ration. Ainsi, pour des quantités d'azote absorbées équivalentes dans les régimes maïs - vermiculite et orge, la valeur est significativement plus faible dans le régime à base d'orge (-9 p. I0o). Par contre, il n'est pas affecté par la présence de vermiculite dans le régime maïs - tourteau de soja. 


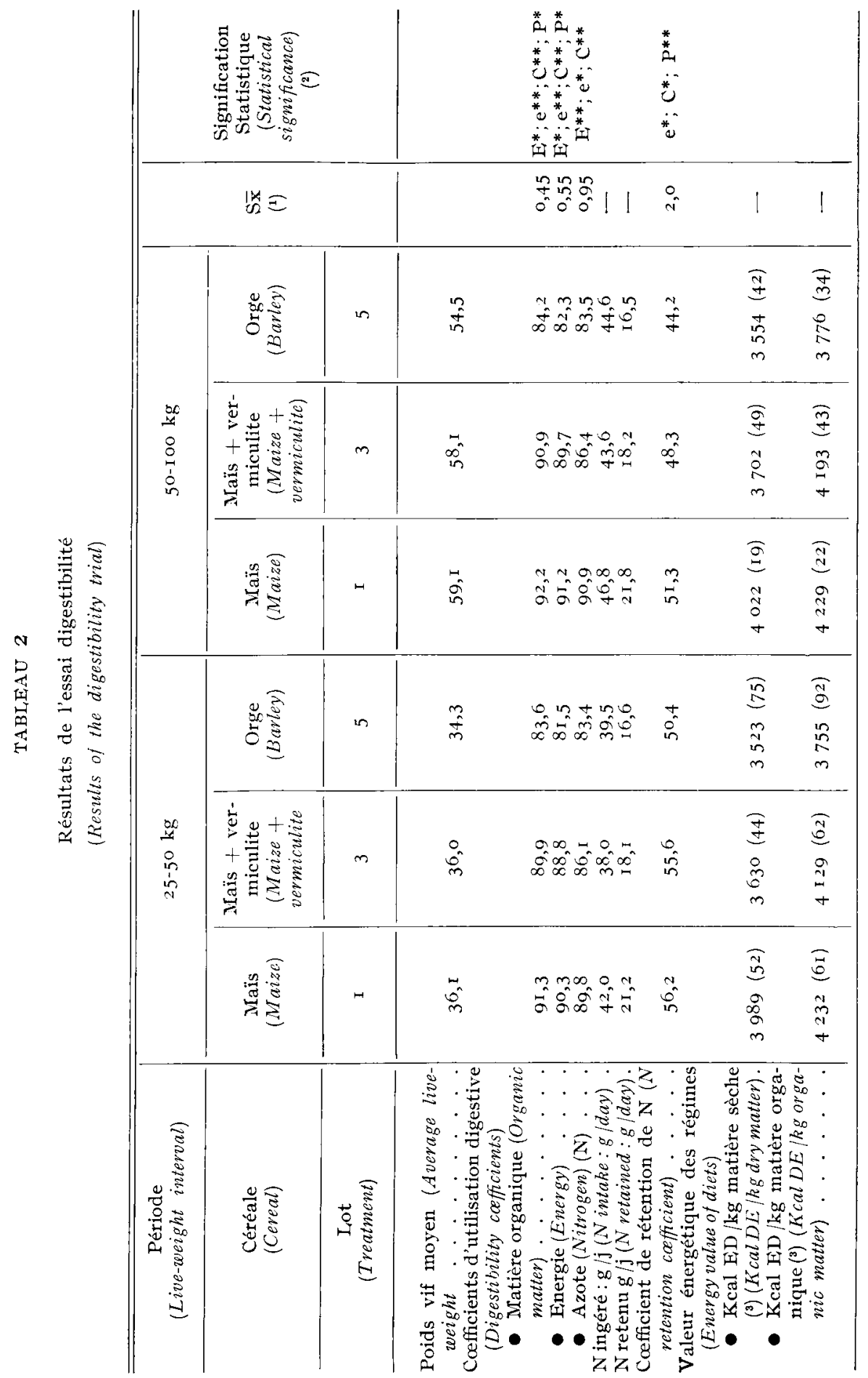


Quoi qu'il en soit, compte tenu d'une digestibilité plus élevée que prévue des régimes à base de maîs et de maïs additionné de vermiculite, la teneur en F.D. de ces derniers reste supérieure à celle des régimes à base d'orge (3.3I contre 3. I6 Mcal E.D. par kg d'aliment pour l'ensemble de l'essai). De même, l'effet dépressif de la vermiculite sur l'utilisation digestive des matières azotées, également plus important que prévu se traduit par une teneur en MAD, relativement à 1 'ĖD, plus faible dans les régimes à base de maïs dilués avec de la vermiculite (lots 3 et 4 ).

\section{II. - Infuence de la concentration en énergie et de la nature des substrats énergétiques sur les performances du porc en croissance-finition (tab1. 3 et 4 )}

Pour l'ensemble des traitements, 1'utilisation de régimes à base d'orge (lots 5 et 6), comparativement à ceux à base de maïs, se traduit par une augmentation de la quantité moyenne journalière d'aliment consommée $(\mathrm{P}<0$,05)et une diminution du niveau correspondant d'ingestion d'E.I. $(\mathrm{P}<0$, IO $)$. La vitesse de croissance n'est cependant pas modifiée, de sorte que les animaux recevant les régimes à base d'orge présentent une adiposité plus faible. De la même façon la dilution du régime maïs - tourteau de soja par la vermiculite provoque une diminution du niveau de consommation d'E.D. $(\mathrm{P}<0,05)$, sans que les vitesses de croissance soient modifiées. Cependant, contrairement à ce que 1'on observe avec les aliments à base d'orge, la dilution du régime maïs - soja n'affecte pas significativement les caractérisriques de composition corporelle.

Dans le cas particulier des régimes normalement pourvus en matières azotées, il semble utile de distinguer les effets de la nature de la céréale (lots I et 5), de la concentration en énergie (lots I et 3 ) et de la nature des substrats énergétiques $(\operatorname{lots} 3$ et 5$)$.

\section{II.I. - Effet de la céréale}

Le remplacement du maïs par l'orge lorsque l'apport de MAD est maintenu constant (g de MAD/Mcal ED), entraîne, pendant la première phase de la croissance $(25-50 \mathrm{~kg})$, une augmentation de la consommation pondérale d'aliment $(+7$ p. IOO), le niveau de consommation d'ED restant cependant inférieur $(6.41$ Mcal ED/jour contre 6,87 ). Cette réduction de l'apport énergétique ne provoque cependant pas de modification de la vitesse de croissance. E,n conséquence, les coûts énergétiques et azotés du gain sont abaissés sensiblement avec les régimes à base d'orge.

Les résultats relatifs à la période totale confirment les observations faites dans l'intervalle de poids $25-50 \mathrm{~kg}$, à savoir une augmentation de $5 \mathrm{p}$. Ioo du niveau de consommation ( $\mathrm{kg}$ d'aliment), une réduction de $4 \mathrm{p}$. roo de la consommation d'ED, un maintien de la vitesse de croissance (7or g/jour contre 70o) et une diminution de $3 \mathrm{p}$. Ioo du coût énergétique ou azoté du gain, lorsque le maïs est remplacé par l'orge.

Dans ces conditions, il n'est pas surprenant de constater que les femelles recevant le régime à base d'orge soient plus maigres $(\mathrm{P}<0,05)$, le rapport longe / bardière étant de 2,60 , contre 2,03 pour les régimes à base de maïs. Cependant, le rendement à l'abattage des animaux du lot 5 est significativement plus faible. 


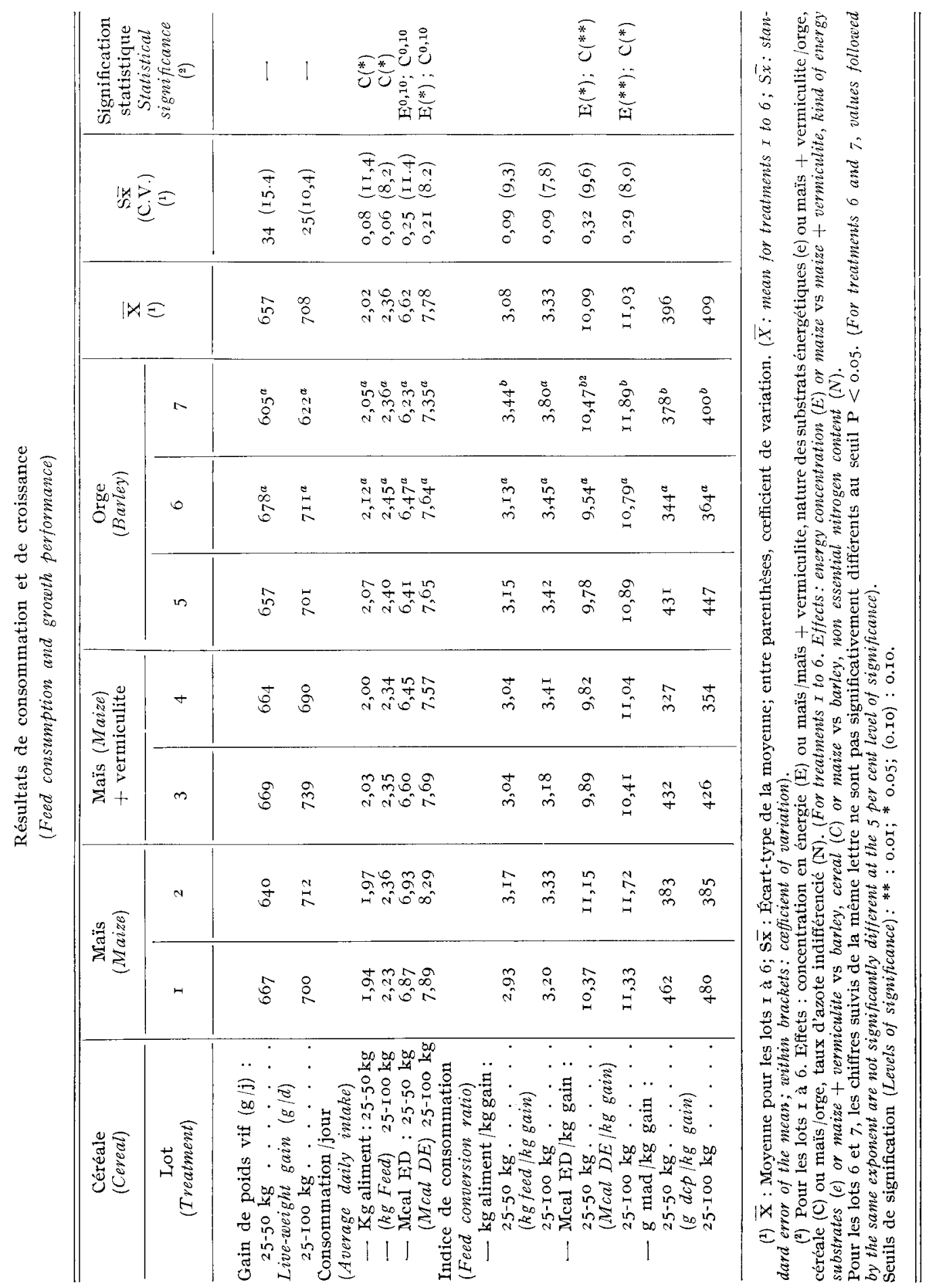




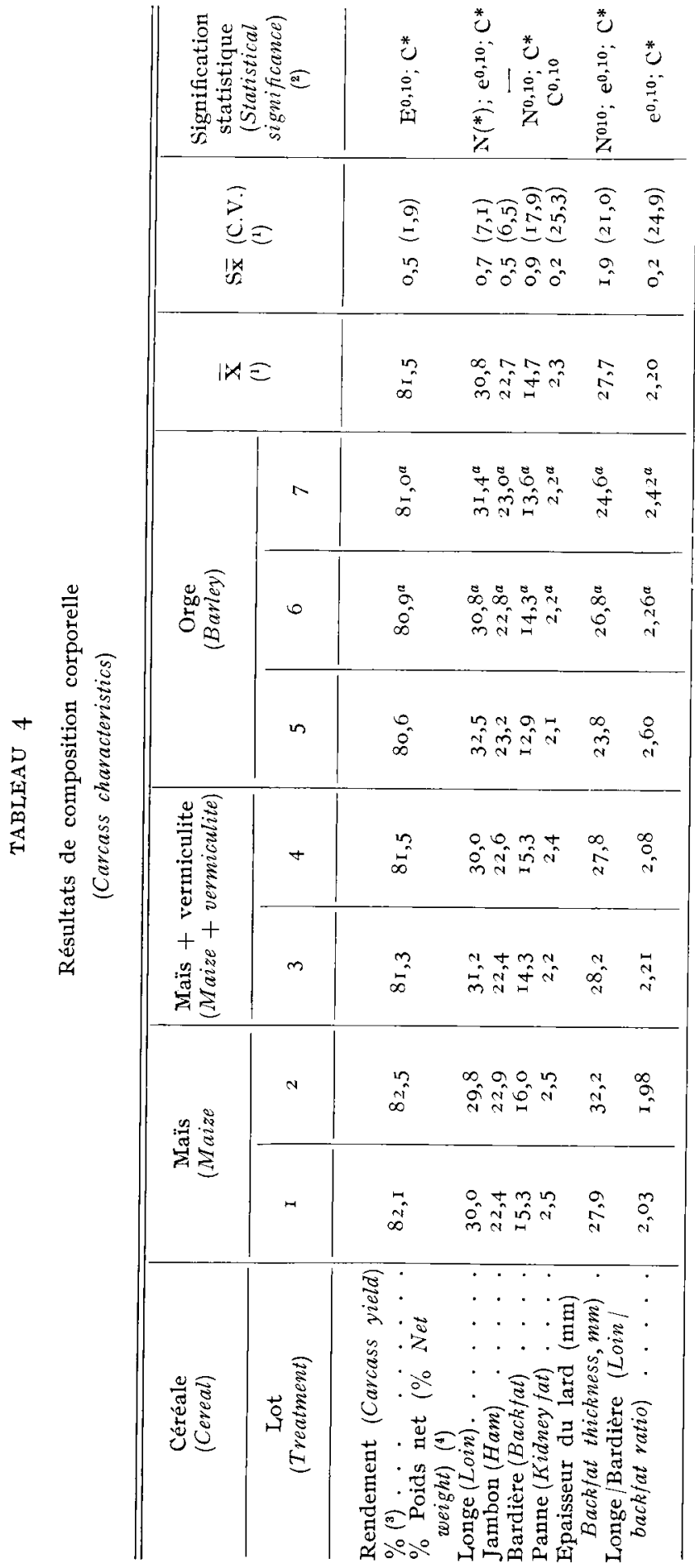




\section{II.2. - Effet de la concentration en énergie.}

L'introduction d'un diluant inerte dans un régime maïs-tourteau de soja, de façon à réduire la concentration en $\mathrm{E} / \mathrm{D}$ d'environ 5 p. Ioo, provoque entre 25 et $50 \mathrm{~kg}$ de poids vif, une légère augmentation du niveau de consommation journalière d'aliment $(2,03 \mathrm{~kg}$ contre $\mathrm{I}, 94)$ mais la consommation d'EID est plus faible $(6,60$ Mcal contre 6,87$)$. De la même façon qu'arec les régimes à base d'orge, la vitesse de croissance n'est pas modifiée. Il s'ensuit une légère diminution des cồts énergétique et azoté du gain.

Comme pour l'intervalle de poids $25-50 \mathrm{~kg}$, la présence de vermiculite dans l'aliment, provoque, entre 25 et Ioo $\mathrm{kg}$ de poids vif, une légère diminution de l'E,D ingérée ( -3 p. 100). Ia vitesse de croissance est toutefois maintenue, ce qui conduit à une réduction sensible des cô̂ts énergétique et azoté du gain (respectivement - 8 et - I I p. Ioo).

Toutefois l'abaissement du niveau d'ingestion d'ED chez les animaux du lot 3, n'entraîne pas de modification importante de leur composition corporelle, bien qu'ils aient tendance à être plus maigres.

En définitive, la réduction de la concentration en $\mathrm{EI})$ du régime maïs-soja, par introduction d'un diluant inerte, la nature des substrats énergétiques restant la même, conduit à un niveau de consommation d' $\mathrm{E} I$ s sensiblement plus faible, sans que la vitesse de croissance et les caractéristiques de composition corporelle soient modifiées significativement.

\section{II.3. - Effet de la nature des substrats énergétiques}

Les régimes distribués aux animaux des lots 3 et 5 étaient prévus isoénergétiques. Cependant, l'aliment offert aux femelles du lot 3 a une concentration en ED sensiblement plus élevée, notamment pendant la période $25-50 \mathrm{~kg}$ de poids vif.

La nature de l'apport énergétique n'affecte pas les niveaux de consommation, que ce soit pendant la période de croissance ou pendant la période totale $(7,65$ contre 7,60 Mcal ED jour). De même, la vitesse de croissance et les coûts énergétique et azoté du gain ne sont pas modifiés significativement. Il en va cependant différemment de la composition corporelle. Ainsi, les femelles recevant le régime maîs - tourteau de soja dilué arec de la vermiculite, présentent un état d'adiposité plus élevé $(\mathrm{P}<\mathrm{O}, \mathrm{IO})$ conme le montrent les écarts de pourcentage de longe, d'épaisseur du lard et du rapport longe/bardière.

En résumé, à la suite d'une dilution énergétique d'un régime équilibré en matières azotées et en acides aminés, le rapport MAD/EI) étant maintenu constant, la quantité d'aliment consommé est accrue tandis que le niveau de consummation d'ED est abaissé. Toutefois, les vitesses de croissance ne sont pas affectées par la réduction de l'apport énergétique. Ea conséquence, les coùts énergétique et azoté du gain pondéral sont sensiblement abaissés. Enfin, pour un même niveau d'apport d'ED, les caractéristiques de composition corporelle sont affectées par la nature des substrats énergétiques. 


\section{III. - Influcnce d'une réduction du taux de matières azotées rééquilibrée: en acides aminés sur les performances de croissance}

Entre 25 et $50 \mathrm{~kg}$ de poids vif, la réduction du taux de matières azotées, lorsque les apports en acides aminés indispensables sont satisfaisants par ailleurs, s'accompagne, avec les régimes maïs-tourteau de soja et orge-tourteau de soja, d'un accroissement, quoique non significatif, du niveau moyen de consommation. Quant aux animaux recevant le régime à base de maîs dilué avec de la vermiculite, leur consommation d'aliment n'est pas modifiée par la réduction du taux azoté global. La vitesse de croissance ne paraît pas affectée de façon significative pendant cette périođe. Il en résulte un effet très limité du taux azoté sur le coût énergétique du gain. Par contre, la diminution du cô̂t azoté du gain avec la réduction du taux azoté apparaît comme hautement significative.

Les observations faites sur 1'ensemble de la période expérimentale (25-100 kg) reflètent celles effectuées entre 25 et $5^{\circ} \mathrm{kg}$ de poids vif. En particulier, la quantité d'aliment consommé est, soit maintenue dans le cas du régime maïs-tourteau de soja dilué avec de la vermiculite, soit légèrement augmentée avec les autres régimes $(+6$ et $+2 \mathrm{p}$. Ioo, respectivement avec les régimes naïs-soja et orgesoja). Toutefois, les animaux élèvent leur niveau de consommation d'énergie digestible uniquement avec le régime maïs-soja $(+5$ p. roo). Comme pour la période đe croissance, la réduction du taux azoté global n'entraîne pas de modification du gain moyen journalier, à l'exception du lot 4 . Cette différence peut être attribuée au niveau de consommation plus faible des animaux de ce lot.

Ces quelques variations dans l'effet de la réduction du taux azoté sur le niveau de consommation et la vitesse de croissance sont à relier à l'augmentation du coût énergétique du gain avec les régimes maîs-tourteau de soja $(+3 \mathrm{p}$. Ioo pour le lot 2 et +6 p. Ioo pour le lot 4). Par contre, avec les régimes orge-tourteau de soja, la réduction de l'apport azoté n'entraîne pas de modification de l'indice de consommation. Dans tous les cas, la réduction du taux azoté s'accompagne d'une diminution hautement significative du cô̂t azoté du gain (- I $9 \mu$. IOO pour l'ensemble des traitements).

Au niveau des caractéristiques de composition corporelle, la diminution du taux azoté se traduit, pour l'ensemble des traitements, par un abaissement significatif $\mathrm{du}$ pourcentage de longe $(\mathrm{P}<0,05)$ et par une augmentation de l'épaisseur du lard $(\mathrm{P}<0, \mathrm{IO})$ et du pourcentage de bardière $(\mathrm{P}<0, \mathrm{IO})$. Toutefois, cet accroissement de l'adiposité est fonction de la concentration en énergie du régime et de la nature des substrats énergétiques. Ainsi, le rapport longe/bardière est réduit de 3 à $6 \mathrm{p}$. Ioo, respectivement avec les régimes maïs et maïs additionné de vermiculite. Cette réduction est plus importante (- I6 p. Ioo) chez les animaux recevant le régime à base d'orge. E,n d'autres termes, la diminution du taux azoté chez des porcs femelles recevant à volonté un régime riche en énergie semble exercer un effet limité sur la composition corporelle, compte tenu de l'adiposité déjà élevée des animaux recevant l'aliment normalement pourvu en matières azotées.

Les résultats du lot 7 font apparaître un effet marqué de l'addition de thréonine dans un régime orge-tourteau de soja ayant une teneur suboptimale en matières azotées (lot 6). Parallèlement à une réduction du niveau de consommation ( -4 p. Ioo pendant la période totale), le gain moyen journalier est abaissé tout au long de la période expérimentale (-I3 p. I00). Il s'ensuit une détérioration sensible de l'indice de consommation (II,89 Mcal ED $/ \mathrm{kg}$ fain contre I0,79). Ce- 
pendant, 1'adiposité des carcasses est réduite. Quoi qu'il en soit, la thréonine constitue, après la lysine, l'acide aminé limitant secondaire d'un régime orgetourteau de soja.

La réduction du taux de matières azotées, accompagnée d'une supplémentation en acides aminés limitants, est donc compensée par une surconsommation d'aliment chez les porcs femilles. Cet effet est surtout sensible avec la ration riche en énergie (maïs-tourteau de soja), mais faible dans le cas de l'association orge-tourteau de soja ou même nulle après dilution des régimes maïs-tourteau de soja par la vermiculite. Cependant, les vitesses de croissance ne sont pas modifiées. Ceci se traduit par une augmentation $d u$ cồt énergétique du gain alors que le coût azoté de ce dernier est réduit de façon très nette. En conséquence, 1'adiposité des carcasses est accrue par la réduction du taux de matières azotées et ce, d'autant plus, que la ration est plus diluée en énergie.

\section{Discussion}

\section{I. - Infuence d'une dilution énergétique du régime sur l'utilisation de l'énergie et des matières azotées}

L'amélioration de l'utilisation digestive de l'énergie chez le porc avec le poids vif (entre 35 et $58 \mathrm{~kg}$ ) est un fait d'observation courante (HENRY et DE WILDE, I 973). De plus, Cunningham, Friend et Nicholson (I962) constatent un accroissement du CUD des constituants cellulosiques de la ration avec le poids vif. L'élévation du CUD $\mathrm{E}$ avec le poids vif devrait donc être plus importante dans le cas d'un aliment relativement riche en substances cellulosiques (régime à base d'orge, par référence à un régime à base de maïs). Cette observation n'est cependant pas confirmée dans le présent essai puisque les écarts constatés sont tout à fait comparables. Le faible éventail, tant dans le poids vif que dans les teneurs en cellulose brute expliquent, en partie, cette absence d'interaction (tabl. 2).

La réduction de la digestibilité de l'ensemble des constituants de la ration à la suite d'une dilution du régime par les constituants cellulosiques ou la vermiculite est conforme à nos résultats obtenus antérieurement (HENRY, I968; HENRY et ETIFNnF, I969). Cependant, l'effet d'un diluant inerte (vermiculite) sur l'utilisation digestive des nutriments semble plus important que dans l'essai réalisé précédemment (HENRY et EiTIENNE, I g69), notamment en ce qui concerne les matières azotées. L'accélération $d u$ transit digestif et l'augmentation des excrétions endogènes avec l'accroissement du volume de l'indigestible expliqueraient cet effet dépressif des constituants membranaires ou de la vermiculite (HENRY et EITIENNE, I969).

\section{II. - Influence de la concentration en énergie et de la nature des substrats énergétiques sur les performances du porc en croissance-finition}

Les résultats de l'essai antérieur (NOBLET et HENRY, I977) avaient fait apparaître une réponse différentielle des mâles castrés et des femelles, à un remplacement du maîs par l'orge dans un régime équilibré en acides aminés. Il en résultait une détérioration des performances de croissance uniquement chez les 
mâles castrés. Chez les femelles, par contre, le niveau d'ingestion, la vitesse de croissance et le cô̂t énergétique du gain étaient comparables, ce qui est aussi le cas dans la présente étude (tab1. 3). Enfin, pour des vitesses de croissance comparables, 1'adiposité des carcasses est réduite de façon significative lorsqu'on utilise le régime à base d'orge conformément aux résultats de GREER et al., (I965).

Les acides gras longs d'origine alimentaire sont déposés préférentiellement dans le tissu adipeux (VAN Es, I 977). Or ceux-ci sont relativement abondants daris les régimes à base de maïs. Les produits terminaux de la digestion tels que les acides gras volatilis et les acides gras courts seraient, au contraire catabolisés et utilisés préférentiellement comme substrat énergétique pour les synthèses, notamment protéiques. La présence de constituants membranaires en quantité non négligeable dans un régime à base d'orge favoriserait donc ce phénomène. En outre, THORBEK (I975) montre que la transformation de l'énergie métabolisable (EM) de l'orge en énergie nette s'effectue avec un rendement plus faible que pour le maïs. Toutes ces observations aboutissent à constater que l'énergie disponible et utilisée pour la formation des dépôts adipeux serait plus importante avec un régime à base de maïs qu'avec un régime à base d'orge, à apport équivalent d'ED ou d'EM.

Cet essai d'explication semble confitmé par l'état d'adiposité moindre des femelles du lot 5 (régime orge-tourteau de soja), comparativement à celles du lot 3 (régime maïs-tourteau de soja dilué par la vermiculite), les niveaux d'ingestion (ED) étant comparables dans les deux lots. En définitive, la réduction de l'état d'adiposité des porcs femelles, lorsque l'orge remplace le maïs, est à relier à une diminution de 1'E,D consommée ainsi qu'à la nature des produits terminaux de la digestion, moins favorables au dépôt lipidique dans le cas de l'orge.

\section{III. - Influence d'une réduction du taux d'azote indifferencie de la ration sur les performances du porc en croissance-finition}

La réduction du taux azoté de la ration, l'apport d'acides aminés indispensables étant maintenu constant, est généralement à l'origine d'une augmentation sensible du niveau de consommation (KATZ et al., I973 : NobLET et HENRy 1977), pour une vitesse de croissance soit comparable (NOBLET et HENRY, I977), soit diminuée (Sharda, Mahax et Wilson, I976). I1 s'ensuit généralement un accroissement du coût énergétique du gain et de l'état d'engraissement. Les résultats rapportés (tableaux 3 et 4 ) confirment ces observations, faites exclusivement à partir de régimes maïs-tourteau de soja.

Cependant, dans l'essai précédent (Noblet et Henry, I977), nous avions noté une interaction entre la concentration en énergie et le taux d'azote indifiérencié sur le niveau de consommation. Une hyperphagie compensatrice plus importante était observée avec le régime riche en énergie (maïs-tourteau de soja) qu'avec le régime orge-tourteau de soja, en particulier chez les mâles castrés, ce qui est en accord avec les résultats obtenus dans le présent travail sur des porcs femelles. Cette observation est retrouvée à la stiite d'une réduction du taux azoté dans un régime à base de mais dilué avec de la vermiculite (lot 4). On peut donc penser que l'encombrement plus important des régimes maïstourteau de soja dilué avec la vermiculite ou orge-tourteau de soja, constitue une limite à une ingestion supplémentaire d'énergie. Toutefois, l'incorporation d'un diluant (substances cellulosiques ou vermiculite) pourrait contrarier la disponibilité des acides aminés, provoquant ainsi une réduction du niveau d'ingestion. 
Enfin, l'effet positif de l'addition de thréonine à un régime orge-tourteau. de soja, à taux azoté suboptimal, montre que cet acide aminé constitue le facteur limitant secondaire de ce type de régime, ce qui est en accord avec les observations de Fulier, Mennie et Croft (i 979).

En conclusion, les résultats de la présente étude montrent que, chez des porcs femelles recevant à volonté des régimes à base de maïs ou d'orge, une réduction du taux global de matières azotées de l'ordre de 25 p. Ioo par rapport aux recommandations habituelles, ne semble pas affecter significativement les performances de croissance, lorsque les besoins en acides aminés indispensables, sont satisfaits. Ceci se traduit par une diminution importante du coût azoté du gain. Toutefois, l'adiposité des carcasses est systématiquement accrue et est plus élevée avec les régimes riches en énergie (maïs-tourteau de soja). Ces régimes, avec le type de porc considéré, conduisent d'ailleurs à l'obtention de carcasses grasses quel que soit le taux d'azote indifférencié. Par contre, l'emploi de régimes à base d'orge, à taux azoté réduit et supplémenté en acides aminés limitants (lysine, thréonine) aboutit, pour des porcs femelles, à des performances de croissance comparables à celles réalisées par des animaux recevant un régime maïs-tourteau de soja à taux azoté normal, qu'il s'agisse du gain moyen pondéral, de l'efficacité alimentaire ou des caractéristiques de composition corporelle.

Ces résultats mettent clairement en évidence le rôle important de la nature des substrats énergétiques sur le besoin en azote indifférencié et sur 1'utilisation nette et la répartition de l'énergie fixée entre les dépôts lipidiques et protéiques. Dans ce sens, des études complémentaires devront être réalisées afin d'étudier l'influence de la nature des substrats énergétiques (lipides, fibres cellulosiques, matières azotées selon l'équilibre en acides aminés...) sur le métabolisme énergétique du porc en croissance, apprécié par la mesure de l'efficacité de l'utilisation de l'énergie et la détermination de la nature des dépôts.

Accepté pour publication en mai 1980.

\section{Summary}

Effects of a reduction in the dietary level of non essential nitrogen in the female growing pig on feed intake, growth performance and carcass characteristics, according to energy concentration and energy substrates

The experiment was performed on 63 female growing pigs between 25 and 1 oo $\mathrm{kg}$ live weight in order to study the infuence of reducing the dietary level of non essential nitrogen on feed intake, growth performance and carcass characteristics, according to energy concentration and the type of energy substrates. Three groups of 9 animals (treatments 1,3 and 5 ) received diets witl protein levels corresponding to the usual recommendations ( $44 \mathrm{~g}$ of digestible crude protein per Mcal Digestible Energy (DE) up to $50 \mathrm{~kg}$ live weight and 39 afterwards), differing either by the energy concentration (Treatment I : maize-soybean meal; Treatment 3 : maize-soybean meal diluted with $6.5 \mathrm{p}$. Ioo of an inert diluent, vermiculite), or by the kind of the energy substrates, for similar DE contents (Treatment 3 and Treatment 5 : barley -soybean meal). For 3 other groups (Treatments 2,4 and 6) of 9 animals in each, the protein level was reduced (- 25 p. IOO) by supplementing with the most limiting anino-acids (lysine, tryptophan, threonine), in order to meet the requirements for growth. One more treatment (Treatment 7) was considered in order to evaluate the effect of threonine supplementation in barley-soybean meal diets, containing suboptimum levels of total protein.

The pigs were individually fed at libitum pelleted diets. They were weighed at two weeks intervals and after slaughter, at $100 \mathrm{~kg}$ live weight; backfat measurements were taken and carcasses evaluated for lean and fat cuts. The results are presented in tables 2, 3 and 4 . 
A complementary trial was carried out on 12 growing male pigs in metabolism crates at two stages ( 35 and $58 \mathrm{~kg}$ live weight) in order to study the effect of energy concentration (by addition of vermiculite) or energy substrates (maize vs barley) on energy and nitrogen digestibilities. The diets used were the same as those of Treatments I, 3 and 5 of the growing trial (Table I)

The addition of vermiculite in a maize-soybean meal diet reduced the digestibility of organic matter, energy and nitrogen $:-.25,-.28$ and -.76 per $\mathrm{p}$. Ioo of vermiculite, respectively. The digestibility coefficients were significantly lower with barley than with maize diets: 81.9 vs 90.7 for energy.

In treatments I, 3 and 5 , the reduction of energy concentration by adding vermiculite or replacing maize by barley, (table 3 ) induced an overconsumption of feed (kg of feed) : +5 and $+7 \mathrm{p}$. Ioo over the $25-$ I $00 \mathrm{~kg}$ live weight interval, respectively. However, the DE consumption was slightly reduced in both cases. The average daily gain (ADG) was not significantly affected by the energy concentration. Consequently, the food conversion ratio (FCR) (Mcal $\mathrm{DF} / \mathrm{kg}$ gain) was reduced when the energy concentration was lower. Carcass adiposity was lower for the barley-soybean treatment but was not reduced significantly when an inert diluent was added to the maize-soybean meal diet (loin/backfat ratio : 2.03, 2.2 I and 2.60 for treatments I, 3 and 5, respectively : table 4).

After a reduction of the nitrogen level, when the limiting essential amino-acids were maintained at the optimum levels for growth, an overconsumption of feed was observed mainly with the high level of energy concentration (maize-soybean meal) : $+6 \mathrm{p}$. Ioo. This effect was smaller for barley diets $(+2$ p. roo) and null for maize diets diluted with vermiculite. The ADG was not affected by the level of non essential nitrogen. Consequently, the FCR ratio was higher for the low nitrogen diets, with the exception of barley diets. Finally, in all treatments, carcass adiposity was increased when the level of non essential nitrogen was reduced (Table 4). But, the variation was greater for barley diets than for maize diets (loin/backfat ratio : 1.98 vs $2.03 ; 2.08$ vs $2.2 \mathrm{I} ; 2.26$ vs 2.60 for maize, maize + vermiculite and barley diets, respectively).

The addition of threonine (treatment $6 \mathrm{vs}$ treatment 7 ) in a low protein barley-soybean meal diet increased intake and ADG in growing females. The FCR was significantly reduced (I0.79 vs II.89 Mcal DE $/ \mathrm{kg}$ gain). Consequently, threonine appears to be the second essential limiting amino-acid after lysine, in a barley-soybean meal diet with a reduced protein level.

\section{Références bibliographiques}

Cunningham H. M., Friend D. W., Nicholson J. W. G., ig62. The effect of age, body weight, feed intake and adaptability of pigs on the digestibility and nutritive value of cellulose. Can J. Anim. Sci., 42, 167-175.

Es A. J. H. VAN, I977. The energetics of fat deposition during growth. Nutr. Motab., 21, 88-104.

FUliek M. F., MENNie I., Crof'Ts R. M. J., I979. The amino acid supplementation of barley for the growing pig. II - Optimal additions of lysine and threonine for growth. Br. J.Nutr., 41, 333 .

Greer S. A. M., Hays V. W., Speer V. C., MCCali J. T., Hammond E. G., i965. Effects of level of corn and barley - base diets on performance and body composition of swine. J.Anim. Sci., 24, I008-IOI3.

HENRY Y., I968. Utilisation comparée des céréales comime seuls aliments du porc pendant la période de finition. Ann. Zootech., 17, I82-197.

HENRY Y., I976. Prediction of energy values of feeds for swine from fiber content. Ist International Symp. Feed Composition, Animal Nutrition Requivements and Computerization of Diets, Utah State Univ. Logan, Utah (U.S.A.).

HENRY Y., E'TIENNE M., I969. Effets nutritionnels de l'incorporation de cellulose purifiée dans le régime du pore en croissance-finition. I - Influence sur l'utilisation digestive des nutriments. Ann. Zootech., 18, 337-357.

HENRY Y, Bourdon D., I973. Utilisation digestive de l'énergie et des matières azotées de la féverole sous forme entière ou décortiquée, en comparaison avec le tourteau de soja. Journées Rech. Porcine en France, 5, 259-264, I.N.R.A. - I.T.P. éd., Paris. 
HFNRY Y., DE WILDS: R., I973. Incorporation de proportions variables de matières grasses (huile d'arachide) dans le régine du poic en croissance-finition I - Influence sur l'utilisation digestive des constituants énergétiques et azotés, et la rétention azotée. Amn. Zootech., 22, I $67-184$.

HEXRY Y., PION R., RERAT A., I976. Protein supplies for pigs and possibilities of reducing protein feeding standards. Wowld Rov. Anim. Prod., 12, 9-32.

KATZ R. S., BAKFR D. H., SASSE C. E., JFNSEN A. H., HARMON B. C., I973. Efficacy of supplemental lysine, methionine and rolled oats, for weanling pigs fed a low protein corn soybean nieal diet. J. Anim. Sci., 37, I 65 -I 688 .

NOMIET J., HExRY Y., I977. Conséquences d'une réduction du taux de matières azotées sur le niveau de consommation et les performances de croissance chez le porc selon l'équilibre en acides anninés et la concentration en énergie du régime. Ann. Zootech., 26, 379-394.

Sharda I). P., Mahan D. C., Wilson R. F., I976. Limiting amino acids on low protein corn soybean meal diets for growing-finishing swine. J. Anim. Sci., 42, I175-1 18 I.

Thorbik G., I975. Studies on energy metabolism in growing pigs. Beveming Statens Husbyrbrugs Fors $\varnothing, 424$, I 57 . 